\title{
ESTUDO DE VIABILIDADE TÉCNICA PARA A REUTILIZAÇÃO DE POLIETILENO TEREFTALATO (PET) RESIDUAL EM SUBSTITUIÇÃO AO AGREGADO MIÚDO NO CONCRETO
}

\section{TECHNICAL VIABILITY STUDY FOR THE REUSE OF POLYETHYLENE TEREPHTHALATE RESIDUAL IN REPLACENT OF THE MEDIUM SAND IN CONCRETE}

\section{Alejandro Salazar Guerra, M.Sc. (UFPR); Elórah Regina Diniz Rocha (Unicuritiba); Juliani Yukie Okabayashi (Unicuritiba)}

\section{Palavras Chave \\ Fibras de PET; Concreto com PET; Construção Sustentável; Reutilização do PET; Concreto Sustentável}

\section{Key Words}

PET Fibers; Concrete with PET; Sustainable Construction; Reuse of PET; Sustainable Concrete

\section{RESUMO}

Esta pesquisa tem como objetivo analisar o comportamento do concreto com substituição parcial do agregado miúdo por Polietileno Tereftalato (PET) e, a sua viabilidade técnica dentro da obra. A partir da revisão bibliográfica e das experiências em laboratório, (seguindo as normas brasileiras), houve a determinação granulométrica da areia fina e média e a fibra de PET afim de verificar as características semelhantes da fibra com um dos respectivos agregados, para posterior substituição deste. Em seguida, ainda em laboratório, foi feita a moldagem, cura, retífica e rompimento dos corpos de prova. Diante dos resultados anotados, observa-se que em pequenas porcentagens de fibras de PET no concreto, há melhora efetiva deste material para seu uso no concreto, porém não sendo recomendado o seu emprego em concretos com funções estruturais.

\section{ABSTRACT}

This research aims to analyze the behavior of concrete with partial replacement of the polyethylene terephthalate (PET) and its technical viability within the work. From the literature review and laboratory experiments (following Brazilian standards), the fine and medium sand and PET fiber were determined in order to verify the similar characteristics of the fiber with one of the respective aggregates, for later replacement of this fiber. Then, in the laboratory, the molding, curing, grinding and breaking of the specimens were done. Considering the results noted, it is observed that in small percentages of PET fibers in the concrete, there is an effective improvement of this material for its use in the concrete however, it is not recommended its use in concretes with structural functions. 


\section{INTRODUÇÃO}

O projeto possui como objetivo analisar o comportamento do concreto com substituição parcial do agregado miúdo por Polietileno Tereftalato (PET), visando assim, determinar se este material é tecnicamente compatível com o concreto e se sua utilização trará benefícios no setor da construção civil.

Tal processo poderá acarretar na redução dos impactos ambientais gerados pelo setor da construção civil, como por exemplo diminuir o consumo de agregados miúdos e conter a quantidade de embalagens plásticas descartadas incorretamente.

Para a realização do projeto, primeiramente foi efetuada uma revisão bibliográfica, analisando as principais características dos materiais a serem utilizados e as normas brasileiras para efetuar os procedimentos de ensaio e estudos sobre os temas abordados.

Após a realização da revisão bibliográfica e o entendimento inicial dos assuntos propostos no projeto, iniciou-se os procedimentos experimentais para a verificação de qual fração do agregado miúdo poderia ser substituído por PET, suas vantagens e desvantagens, bem como as características apresentadas após aplicação e cura da composição.

\section{REVISÃO BIBLIOGRÁFICA}

O Conselho Internacional da Construção (CIB), aponta a indústria da construção civil como o setor de atividades humanas que mais consome recursos naturais e utiliza energia de forma intensiva, gerando consideráveis impactos ambientais (OLIVEIRA, 2013). Para se ter uma ideia, no Brasil para a produção de concreto e argamassa, no ano de 2000, o consumo era de 220 milhões de toneladas de recursos naturais como areia, brita, calcário, entre outros (JOHN, 2000).

Segundo Milhorance (2016), o Brasil, junto de outros quatro países (China, Índia, EUA e Turquia), está entre os principais produtores de cimento do mundo. Juntos, são responsáveis por $70 \%$ desta produção. 0 grupo também está entre os grandes mineradores de areia, sendo $80 \%$ desta extração, destinada a indústria da construção civil, como por exemplo para a produção de concretos e vidros.

Além dos impactos referentes ao consumo de matéria e energia, há aqueles associados à geração de resíduos sólidos, líquidos e gasosos. A construção civil gera hoje uma grande quantidade de resíduos, causando sérios problemas relacionados ao meio ambiente, fazendo-se necessário identifica-los para buscar soluções cabíveis e eficientes para que estes sejam minimizados, evitando problemas futuros mais sérios.

Na busca de minimizar os impactos ambientais provocados pela construção, surge a iniciativa para uma construção sustentável. Partindo disso, vem crescendo a ideia do uso de materiais reciclados na construção civil como as fibras de PET, provenientes de embalagens plásticas. Além de reduzir tais impactos, a utilização das mesmas poderá diminuir o número de garrafas presentes nas ruas. Se calcularmos a produção de apenas $1 \mathrm{~m}^{3}$ de concreto, utilizamos cerca de $760 \mathrm{~kg}$ de areia. Substituindo apenas $5 \%$ dessa areia por fibras de PET, seriam aproximadamente 890 garrafas de $500 \mathrm{ml}$ retiradas das ruas para utilização em um material inerte, o que trará benefícios imediatos ao meio ambiente. Para se ter uma ideia de grandeza em 2012, por exemplo, as concreteiras instaladas no país produziram cerca de 51 milhões de $\mathrm{m}^{3}$ (OLIVEIRA, 2013).

Segundo o $9^{\circ}$ censo da ABIPET (2013), em 2011 foram produzidas cerca de 9 bilhões de garrafas, sendo destas apenas 5,3 bilhões recicladas em 2012. No Brasil, no mesmo ano, dos 5570 munícipios brasileiros (IBGE, 2013) apenas 766 fizeram a coleta seletiva (ABIPET, 2013). Esse número representa somente $15 \%$ dos municípios do país com participação na coleta.

Entre as aplicações do PET reciclado atualmente, $46 \%$ são destinados para Botle to Botle (o qual trata-se de uma embalagem produzida a partir de garrafas pós-consumo recicladas), $25 \%$ para área têxtil, $18 \%$ outros e $11 \%$ área automotiva (ABIPET, 2013). Seu uso na construção civil ainda não contribui com uma porcentagem significativa, no entanto, vários estudos já foram realizados para utilização de fibras desse material, como alternativa para substituição parcial do agregado miúdo no concreto.

Em estudos realizados para a implantação das fibras de PET no concreto, observou-se na pesquisa de Betioli et al (2004) sua durabilidade em materiais a base de cimento, o qual verificou um melhor comportamento pós-fissuramento e uma resistência maior a carga (por alguns minutos após a falha), sem que ocorresse desintegração completa.

Maragon (2003) afirma que houve uma maior tenacidade com o uso das fibras de PET. O mesmo afirma ainda que o uso destas geraram um concreto mais dúctil, propriedade de um concreto flexível, elástico e maleável, onde experimenta deformações inelásticas sem a perda de sua capacidade resistente, causando uma deformação antes da ruptura completa.

Meneses (2011) realizou o estudo da utilização destas fibras em concretos submetidos a altas temperaturas, 
concluindo que tal utilização pode retardar o risco de colapso de estruturas pela formação de uma rede de canais que facilitam a fuga do vapor d'água, reduzindo a poropressão no interior do elemento estrutural. Silva (2013) comprovou tal efeito em seus estudos analisando a resistência a compressão e porosidade de concreto quando submetidos a elevadas temperaturas, obtendo resultados como a não ocorrência do efeito spalling nos corpos de prova que possuíam as fibras.

Várias pesquisas concluíram que o aumento das porcentagens de fibras de PET em substituição parcial ao agregado miúdo, causavam perda na resistência a compressão do concreto. Almeida et al (2004) indica uso de teores menores que $50 \%$. Canellas (2005), reforça essa tese, pois utilizou em sua pesquisa teores de 10\%, 30\%, 50\% e $100 \%$ e obteve melhores resultados nos teores entre $10 \mathrm{e}$ $30 \%$. Cândido, Barreto e Cabral (2014) que utilizaram teores de $15 \%, 30 \%$ e $45 \%$, obtiveram seus melhores resultados com o teor de $15 \%$.

\section{MATERIAIS E MÉTODOS}

\subsection{Caracterização dos materiais}

Inicialmente foi realizado um estudo comparativo dos perfis granulométricos entre os agregados miúdos (areia fina e média) e as fibras de PET, afim de estabelecer qual deles as fibras mais se assemelham, em granulometria, para a posterior substituição.

O teste foi realizado de acordo com a NBR 248 Determinação da composição granulométrica, com o objetivo de padronizar as areias média e fina, além das fibras de PET. Para isso, utilizou-se do agitador mecânico, amostras devidamente secas e as seguintes peneiras $(\mathrm{mm}): 4,5$ $-4-3,35-2,8-2,36-1,18-0,6-0,3-0,15-0,075-0,045$ e fundo, todas posicionadas em ordem crescente da base para o topo.

Com esse teste foi estabelecida a faixa granulométrica da areia fina caracterizada com $0,045 \mathrm{~mm}-0,3 \mathrm{~mm}$ e a areia média com $0,3 \mathrm{~mm}-1,18 \mathrm{~mm}$. As fibras de PET obtiveram uma curva granulométrica com características próximas a granulometria da areia média, conforme pode ser observado no Gráfico 1(Curva Granulométrica Areia Média e fibras de PET) e Gráfico 2 (Curva Granulométrica Areia Fina e fibras de PET).
Gráfico 01 - Curva Granulométrica Areia Média e fibras de PET.

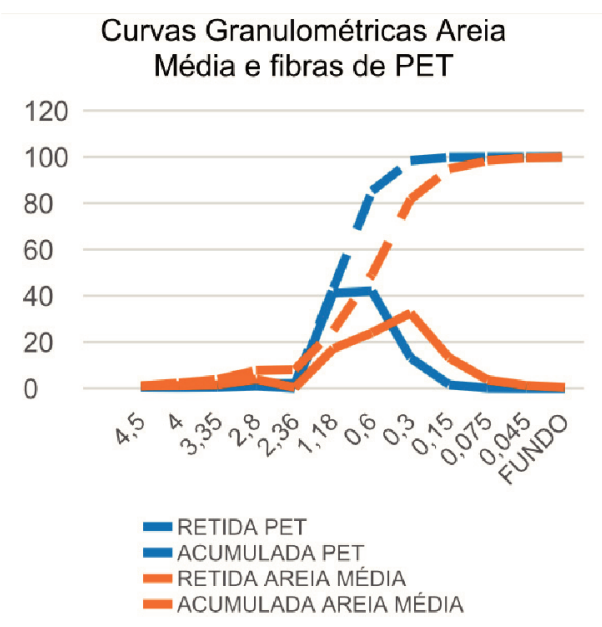

Fonte: Elaborado pelo autor

Gráfico 02 - Curva Granulométrica Areia Fina e fibras de PET

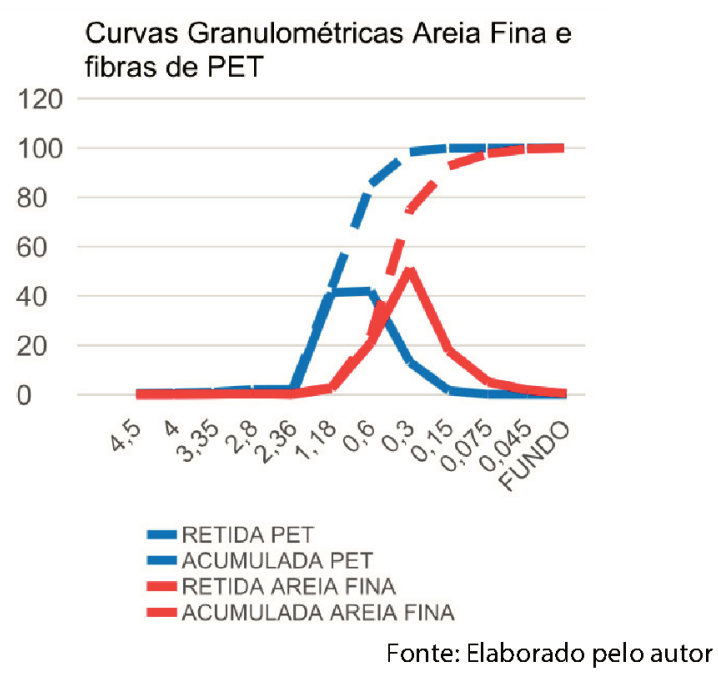

Através do ensaio de granulometria, foi possível determinar qual dos dois agregados miúdos (utilizados no traço), seria substituído pelas fibras de PET. Escolhendo o agregado com maior semelhança granulométrica quando comparada as fibras.

Observando os gráficos pode-se perceber, uma maior similaridade entre a areia média e as fibras PET. E devido tal semelhança determinou-se que, as fibras iriam substituir porcentagens no volume da areia média.

O aglomerante utilizado foi o cimento Portland - CP II Z - 32 e como agregado graúdo, utilizou-se da pedra brita 1, peneirada em granulometria de 9,5 mm - $19 \mathrm{~mm}$.

\subsection{Moldagem, cura, retífica e rompimento dos corpos de prova}

Iniciou-se os procedimentos para a moldagem dos corpos de prova de concreto referencial (CR) - convencional - e dos 
corpos de prova com substituição da areia média pelas fibras de PET. As substituições testadas, em percentuais, foram de $20 \%, 30 \%$, e $50 \%$ do volume da areia média. Estes testes foram efetuados seguindo as normativas brasileiras: NBR 9479 - Câmaras úmidas e taques para cura de corpos-de-prova de argamassa e concreto, NBR 5739 - Ensaio de compressão de corpos-de-prova cilíndricos e NBR 5738 - Procedimento para moldagem e cura de corpos-de-prova.

Os materiais foram pesados de acordo com o traço calculado com resistência a compressão esperada de $35 \mathrm{MPa}$ aos 28 dias, no entanto foram realizados testes até os 21 dias, os quais já alcançaram o valor proposto. Para o corpo de prova de referência (CR), utilizou-se os seguintes materiais: $4,8 \mathrm{~kg}$ de cimento CP- II- Z- 32; 4,224 kg areia média; $504 \mathrm{~g}$ de areia fina; 11,57 kg de pedra 1 e 2,3 I de água, sendo um traço de 1:0,88: 0,105: 2,41:0,48.

Após a pesagem de todos os materiais, iniciou-se a moldagem dos corpos de prova, sendo eles introduzidos na betoneira na seguinte ordem: pedra 1; uma fração da água; cimento; areias/PET (quando necessário) e outra fração da água. Este processo foi realizado em um período de 10 a 15 minutos, com paradas para a remoção de concreto preso ao fundo da betoneira. Quando atingido uma consistência homogênea, o concreto foi introduzido aos moldes dos corpos de prova -
Pôde-se perceber pelas curvas obtidas, que ao aumentar a porcentagem de fibras de PET no concreto sua resistência diminui, como foi verificado no referencial teórico (GRAFICO 3).

No entanto, notou-se também, que os corpos de prova com adição de fibras de PET mostraram-se mais tenazes, conforme Maragon (2013) havia descrito em seus experimentos, observando que os concretos com adição das fibras após sua ruptura não se desintegraram completamente, ficando com seus fragmentos "presos" ao corpo de prova, como pode ser visualizado nas figuras (01) e (02), onde mostram um corpo de prova com adição, e um sem adição das fibras.

Figura 01 - Concreto com adição 30\% de PET.

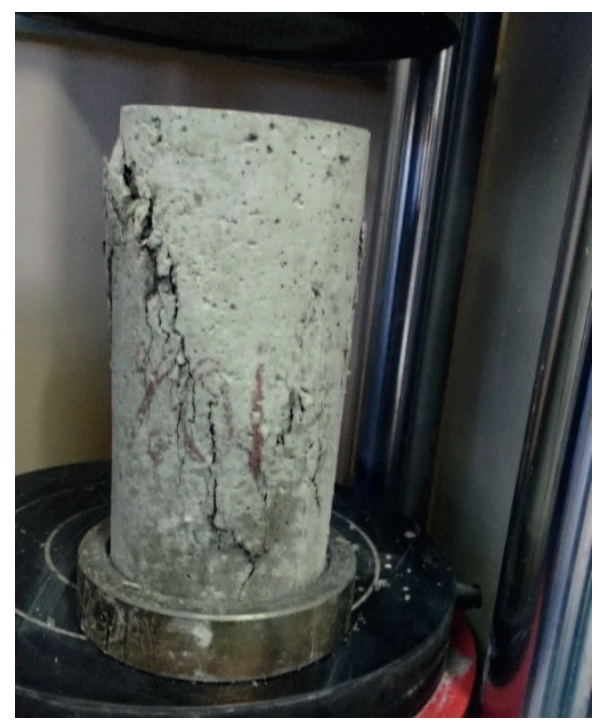

Fonte: Elaborado pelo autor

\section{RESULTADOS OBTIDOS}

Após o rompimento dos corpos de prova na prensa hidráulica, obteve-se os seguintes dados das resistências a compressão axial dos corpos de prova ensaiados.

Gráfico 03 - Resistência a Compressão Axial Mpa/Dias

\begin{tabular}{|c|c|c|c|}
\hline & 7 & 14 & 21 \\
\hline CR & 28,67 & 35,98 & 38,05 \\
\hline $20 \%$ & 26,43 & 30,97 & 35,93 \\
\hline $30 \%$ & 22,56 & 27,64 & 31,45 \\
\hline $50 \%$ & 19,58 & 23,98 & 28,54 \\
\hline
\end{tabular}

Fonte: Elaborado pelo autor
Figura 02 - Concreto sem adição de PET (CR).



Fonte: Elaborado pelo autor 


\section{CONCLUSÕES}

Ao analisar os resultados obtidos após o rompimento dos corpos de prova, pode-se concluir que ao aumentar a adição do teor de fibras de PET, as propriedades mecânicas do concreto são alteradas. Exemplo disso, foi observada a redução da resistência a compressão axial nas duas amostras mencionadas, não sendo o uso destas fibras indicado, (nos percentuais pesquisados), para concretos com funções estruturais.

Apesar disso, o concreto em estudo - com adição de fibras de PET - pode ser viável sob outros aspectos, uma vez que foi observado o aumento de sua tenacidade, o que reduz o efeito de colapso da estrutura de concreto.

Além de observar benefícios ligados as propriedades mecânicas do concreto, pode-se observar também, benefícios ligados ao meio ambiente, como a redução do consumo de areia, diminuindo os impactos ambientais gerados por essa extração, além de minimizar a quantidade de embalagens PET das ruas e depósitos, pois quando estas são descartadas de maneira incorreta, podem acarretar em enchentes e alagamentos.

Estudos relacionando o percentual máximo que o concreto pode receber a adição de fibras de PET, em substituição ao agregado miúdo, são de grande valia, uma vez que podem impulsionar o consumo deste material e aumentar os incentivos para o reaproveitamento e reciclagem, ajudando no bem-estar da sociedade e reduzindo a poluição em nosso planeta.

\section{AGRADECIMENTOS}

Agradecemos ao Centro Universitário Unicuritiba, ao coordenador Eng. Renato Braga Coelho Neto do curso de Engenharia Civil e ao Senhor Andre Barriachello da MAC PET.

\section{REFERÊNCIAS}

ALMEIDA, Marconi Oliveira de et al. Uso de areia de PET na fabricação de concretos. In: Congresso Brasileiro de Ciência e Tecnologia e Resíduos e Desenvolvimento Sustentável 2004, Florianópolis, Livro de Resumos, São Paulo, ICTR, 2004. P.39.

ASSOCIAÇÃO BRASILEIRA DA INDÚSTRIA PET (ABIPET). $9^{\circ}$ Censo da Reciclagem de PET no Brasil. Disponível em: $<$ http://www.abipet.org.br/index.html?method=mostrarDownloads\&categoria.id=3>. Acesso em: 16 fev. 2016.

\section{ASSOCIAÇÃO BRASILEIRA DE NORMAS TÉCNICAS. NBR} 9479 - Câmaras úmidas e taques para cura de corpos-de-prova de argamassa e concreto. Rio de Janeiro, 1994.
. NBR 248 - Determinação da composição granulométrica. Rio de Janeiro, 2003.

NBR 5739 - Ensaio de compressão de corpos-de-prova cilíndricos. Rio de Janeiro, 2007.

NBR 5738 - Procedimento para moldagem e cura de corpos-de-prova. Rio de Janeiro, 2015.

BETIOLI, Andrea Murilo et al. Degradação de fibras de PET em materiais à base de cimento portland. Anais: $X$ Encontro Nacional de Tecnologia do Ambiente Construído. São Paulo, 2004.

CÂNDIDO, Luis Felipe; BARRETO, José Maurício Lima; CABRAL, Antônio Eduardo Bezerra. Avaliação de blocos de concreto produzidos com PET reciclado. Anais: XV Encontro Nacional de Tecnologia do Ambiente Construído. Maceió, 2014.

CANELLAS, Susan Sales; D'Abreu, José Carlos. Reciclagem de PET, visando a substituição de agregado miúdo em argamassas. Dissertação - Departamento de Ciência dos Materiais e Metalurgia - Pontifícia Universidade Católica do Rio de Janeiro, Rio de Janeiro, Brasil, 2005.

INSTITUTO BRASILEIRO DE GEOGRAFIA E ESTATÍSTICA. Censo IBGE 2013. Disponível em: <http://www.ibge. gov.br/home/estatistica/economia/perfilmunic/2013/>. Acesso em: 21 out. 2016.

JOHN, Vanderley Moacyr. Reciclagem de resíduos na construção civil. 113 f. Tese (Doutorado) - Departamento de Engenharia de Construção Civil - Escola Politécnica da Universidade de São Paulo, São Paulo, 2000.

MARAGON, Ederli. Aspectos do comportamento e da degradação de matrizes de concreto de cimento portland reforçados com fibras provenientes da reciclagem de garrafa pet. Disponível em: <http://www. projetos.unijui.edu.br/petegc/wp-content/uploads/tccs/ tcc-titulos/2003/Aspectos_do_Comportamento_e_da_ Degradacao_de_Matrizes_de_Concreto_de_Cimento Portland_Reforcados_com_Fibras_PET>. Acesso em: 16 fev. 2016.

MENESES, Ilzenete Andrade. Avaliação de concreto com adição de fibras de PET submetido a altas 
temperaturas. 90 f. Dissertação (Mestrado) - Programa de Pós-Graduação em Engenharia Civil - Universidade Federal do Rio Grande do Norte, Natal, 2011.

MILHORANCE, Flávia. Base da construção civil, areia é um dos recursos mais valiosos e explorados do mundo. $\mathbf{O}$ Globo. Disponível em: < http://oglobo.globo.com/sociedade/sustentabilidade/base-da-construcao-civil-areia-um-dos-recursos-mais-valiosos-explorados-do-mundo-14960573>. Acesso em: 20 out. 2016.

OLIVEIRA, Marta. Pesquisa inédita e exclusiva revela cenário do mercado brasileiro de concreto. Associação Brasileira de Cimento Portland, São Paulo, ago. 2013. Disponível em: < http://www.abcp.org.br/cms/imprensa/noticias/pesquisa-inedita-e-exclusiva-revela-cenario-do-mercado-brasileiro-de-concreto/ >. Acesso em: 07 nov. 2016.

SILVA, Janaina Salustio da. Estudo de concretos de diferentes resistências à compressão quando submetidos a altas temperaturas sem e com incorporação de fibras de politereftalato de etileno (PET). $148 \mathrm{f}$. Dissertação (Mestrado) - Programa de Pós-Graduação em Engenharia Civil - Universidade Federal do Rio Grande do Norte, Natal, 2013. 